\title{
Metodologia ADDIE para CONSTRUÇÃo de PROduto EdUCACIONAL EM SAÚde Para FENILCETONÚRIA
}

Monique Oliveira Poubel (monique.poubel@escs.edu.br) - Escola Superior em Ciências da Saúde, ESCS.

Adriana Haack de Arruda Dutra (adrianahaack@escs.edu.br) - Escola Superior em Ciências da Saúde, ESCS.

\section{RESUMO}

A fenilcetonúria é uma doença rara, autossômica recessiva, caracterizada por acúmulo do aminoácido fenilalanina (FAL) no sangue e outros tecidos. O conhecimento sobre a doença e o seu tratamento constituem os pilares do auto manejo responsável da Fenilcetonúria. Há escassez de materiais educacionais padronizados para o paciente sobre a doença e sua compreensibilidade também não foram investigadas sistematicamente. Na literatura há insuficiência de referências práticas publicadas sobre o desenvolvimento de produtos educacionais em saúde para a fenilcetonúria. O conteúdo do produto educacional deve ser maleável como todo os processos de educação e aberto para acolher as diferentes demandas dos usuários em saúde, o modelo de design instrucional ADDIE possibilita esta construção devido a sua característica genérica e flexível, seu uso centrado no paciente e sua coerência com as teorias de aprendizagem possibilitam a construção de um instrumento educacional para ser utilizado pela equipe multiprofissional de assistência à fenilcetonúria.

Palavras-chave: Desenho Instrucional; Educação Nutricional; Fenilcetonúria; Promoção da saúde. 


\section{INTRODUÇÃO}

A fenilcetonúria (PKU; OMIM \# 261600) é um erro inato do metabolismo (EIM), uma doença rara, autossômica recessiva, caracterizada por acúmulo do aminoácido fenilalanina (FAL) no sangue e outros tecidos. A causa mais frequente do aumento de FAL sérica são as mutações no gene que codifica a fenilalanina hidroxilase (PAH) (PAH: EC 1.14.16.1), que interfere exclusivamente na hidroxilação hepática da fenilalanina, pela deficiência da enzima PAH, entretanto $1-2 \%$ dos casos são ocasionados pela deficiência no cofator tetrahidrobiopterina (BH4) (na enzima dihidro- biopterina redutase DHPR, EC 1.6.99.7) ou em enzimas envolvidas na biossíntese e reciclagem do cofator (BLAU et al., 2018). A incidência de PKU varia amplamente no mundo. Na Europa, a prevalência estimada é de 1:10.000 nascidos vivos, na Turquia, de 1 em cada 400 nascidos vivos, devido à elevada consanguinidade (VAN WEGBERG et al., 2017). Na América Latina e no Brasil, incidências 1: 23.500 (variação de 1:12.400-1:161.700) e 1: 23.600 nascidos vivos foram estimados, respectivamente (BORRAJO, 2012).

A Triagem Neonatal para fenilcetonúria, seguida do tratamento com uma dieta restrita em fenilalanina, que controla as concentrações sanguíneas desse aminoácido e resulta na redução da maioria das complicações neuropsicológicas (BILDER et al., 2016), entretanto, o seguimento e a manutenção da dieta restrita é árdua, especialmente entre adolescentes e adultos (BLAU; VAN SPRONSEN; LEVY, 2010).O tratamento da deficiência de PAH deve ser realizado por toda a vida e a manutenção de um nível de FAL de 120 a $360 \mu \mathrm{mol} / \mathrm{L}$, em pacientes tratados, é recomendado para todos, em todas as idades (VOCKLEY; ANDERSSON; ANTSHEL et al., 2014).

A base do tratamento da fenilcetonúria consiste em uma dieta restrita de proteínas naturais, pois esse aminoácido tem presença preponderante em alimentos com alto teor proteico, assim deve-se realizar a exclusão de leguminosas, da maioria dos cereais e de alimentos ricos em proteínas de alto valor biológico (carnes, peixes, ovos e laticínios), devese realizar também a ingestão de um complemento de aminoácidos isento de FAL e alimentos com pouca proteína (BRASIL, 2011; SINGH et al., 2014). Para que estas pessoas e suas famílias tenham conhecimento da doença e do manejo adequado dos alimentos, diante da necessidade da restrição dietética, deve haver processos educacionais humanizados e efetivos, que permitam que uma escolha alimentar apropriada. 
A assistência à saúde é reconhecida pela abordagem socioambiental como fundamental na determinação do processo saúde-doença, sugerindo a reorientação dos serviços e sistemas de saúde para o fortalecimento de ações de promoção da saúde. Uma das estratégias-chave do movimento de Promoção à Saúde é o conceito de empowerment ou empoderamento. Por meio do empoderamento, a Promoção à Saúde possibilita aos usuários um aprendizado que os torne capazes de administrar as limitações impostas por eventuais enfermidades, sugerindo que estas ações devam ser realizadas em vários locais, na escola, no domicílio, no trabalho e nos espaços comunitários (BRASIL, 2002; POLAND; KRUPA; MCCALL, 2009).

$\mathrm{Na}$ literatura, há insuficiência de referências práticas publicadas sobre o desenvolvimento de produtos educacionais para fenilcetonúria e uma dificuldade em identificar inovações e ações exitosas em Educação Nutricional para motivar os profissionais de saúde a desenvolver e utilizar produtos educacionais adaptados à realidade brasileira que promovem escolhas alimentares adequadas e saudáveis.

O planejamento de uma inovação educacional em saúde depende dos objetivos, do alcance populacional pretendido, da avaliação de sua eficácia e da extensão em que é implementada com níveis elevados de integridade e fidelidade. Revisões sistemáticas recentes assinalam a importância do planejamento instrucional na efetividade do treinamento em saúde com simulações utilizando diretrizes instrucionais (COOK et al., 2013). O desenvolvimento de soluções instrucionais deve seguir um método sistemático para elaboração de programas ou materiais de educação e treinamentos para melhorar o conhecimento do paciente e de sua família.

O desenho instrucional (DI) é um recurso de planejamento destinado a garantir o desenvolvimento de atividades de aprendizagem de forma sistemática e coerente. O conceito de Instructional System Design (ISD) existe desde o início dos anos 1950, o ADDIE surgiu pela primeira vez em 1975. Foi criado pelo Center for Educational Technology na Florida State University para o Exército dos EUA e depois rapidamente adaptada por todas as Forças Armadas dos EUA (WATSON, 1981). O ISD, também conhecido como ADDIE, é um dos modelos sistêmicos mais conhecidos, que compreende cinco fases: 1) análise; 2) desenho; 3) desenvolvimento; 4) implementação e 5) avaliação, este modelo permite a visão geral do processo de aprendizagem e as correções necessárias para garantir a sua efetividade (MELO 
et al., 2018).

O modelo de design instrucional ADDIE é genérico, flexível, deve ser centrado no paciente para a realização do planejamento um produto educacional em saúde e é coerente com as teorias de aprendizagem eletrônica, uma exigência atual neste período de pandemia de COVID-19. Assim, o presente artigo apresenta o desenho metodológico ADDIE para a construção de um instrumento educacional para ser utilizado pela equipe multiprofissional de assistência à fenilcetonúria.

\section{METODOLOGIA}

A coleta de dados foi realizada no período de 02 de junho a 04 de agosto de 2021, nas bases de dados Literatura Latino-Americana e do Caribe em Ciências da Saúde (LILACS), Scientific Eletrônic Library Online (SCIELO), SCOPUS e National Library of Medicine (PUBMED). Foi definido como critério de inclusão: artigos publicados entre os anos de 2011 e 2021, pois levantamentos iniciais identificaram que há escassez de artigos que descrevem com maior detalhamento as práticas de educação em saúde em fenilcetonúria realizadas pelos profissionais da saúde. Quanto aos descritores em ciências da saúde, foram incluídos neste estudo artigos que apresentassem descritores como: Educação Alimentar e Nutricional, Comunicação em Saúde, Fenilcetonúrias suas combinações e variantes em inglês ("Food and Nutrition Education" or "Health Communication" and "Phenylketonurias"). Não foi limitado o idioma na tentativa de obter quantidade relevante de referencial teórico. Como resultados, foram obtidos 01 artigo na LILACS e 01 artigo na SciELO, cujo tema não estava de acordo com este estudo e que foi publicado também em ano anterior a 2011. A pesquisa na PUBMED resultou em 10 artigos, dos quais 2 estavam de acordo com os objetivos do estudo e na SCOPUS resultou em 31 artigos, e apesar de não ter como tema a fenilcetonúria foi selecionado um artigo, utilizado na discussão devido a temática das doenças raras e a importância de diferentes fontes de informação. Foram excluídos estudos relacionados à triagem neonatal, aspectos culturais, Eixo intestino-cérebro, sapropterina e alimentos com baixo teor de proteína. Devido ao número reduzido de artigos encontrados nas bases de dados LILACS, SciELO, PUBMED e SCOPUS, denota-se a reduzida disponibilidade de estudos relacionados ao tema. Dessa forma, ao final da pesquisa nas bases de dados citadas, foram encontrados 43 artigos, porém, apenas 03 apresentavam o tema educação em saúde nas práticas dos profissionais para o tratamento de doenças raras. 
Além da revisão da literatura para o levantamento de conhecimentos específicos que atendam à complexidade do fenômeno saúde-doença por meio da consulta nas bases de dados, foi realizada a análise dos dados das consultas ambulatoriais de pacientes adultos e adolescentes de maio 2020 a junho de 2021, com enfoque nas principais dificuldades e dúvidas para adesão ao tratamento dos pacientes fenilcetonúricos. Estes dados identificaram os principais obstáculos à adesão à terapêutica nutricional para construção da cartilha de promoção da educação nutricional para os Fenilcetonúricos.

Dúvidas, expectativas e necessidades educacionais identificadas pelos pacientes e cuidadores foram registradas durante as consultas de rotina para avaliar o conhecimento atual sobre a doença e as dificuldades do tratamento que serão abordados no design instrucional da cartilha educativa, norteando a elaboração dos tópicos e do conteúdo principal. O conteúdo do produto deve ser maleável como todo os processos de educação, flexíveis e abertos para acolher as diferentes demandas dos usuários em saúde.

\section{RESULTADOS E DISCUSSÃO}

Encontrar informações confiáveis sobre uma das mais de 7.000 doenças raras é um grande desafio para as pessoas afetadas. As dificuldades de acesso às informações são comuns e pouco se sabe sobre o uso de fontes de informação para diferentes doenças raras, como os usuários classificam essas fontes de informação com base em suas experiências e como o uso e a importância de essas fontes de informação mudam com o tempo (LITZKENDORF et al., 2020).

Cuidadores e pacientes podem encontrar muitas barreiras para a adesão ao tratamento, a maioria das quais não são exclusivas da PKU. Essas barreiras incluem, por exemplo, dificuldades cognitivas (não é capaz de entender as recomendações e o seu tratamento) e educacionais (falta de informações e justificativa para recomendações de tratamento) (BANNICK et al., 2015). Na prática ambulatorial observa-se que o conhecimento sobre a doença e o seu tratamento constituem os pilares do auto manejo responsável da Fenilcetonúria. Não há muitos materiais padronizados de educação do paciente sobre a doença e sua compreensibilidade não foram investigadas sistematicamente na literatura.

A complexidade do EIM (Erro Inato do Metabolismo) torna difícil a compreensão da sua fisiopatologia, herança e fundamentos terapêuticos para os pacientes e 
cuidadores. Especialmente os pacientes que chegam à adolescência e à idade adulta podem ter apenas um conhecimento limitado de sua condição, uma vez que os cuidados médicos muitas vezes são administrados em sua totalidade pelos pais (ZELTNER et al., 2019).

Nesta direção, o desenvolvimento de uma cartilha educacional se reveste de importância, seu conteúdo e apresentação (Quadro 01) deve fornecer informações oportunas, relevantes e acessíveis com o objetivo de apoiar os indivíduos a assumirem um papel ativo em sua própria saúde encorajando os a atingir a meta terapêutica de manter os níveis de fenilalanina normais e associá-la à qualidade de vida. As intervenções dietéticas que serão apresentadas (alimentos, fonte de proteínas, teor de aminoácido etc.) se relacionam diretamente com as necessidades e objetivos do tratamento.

Quadro 01 - Características da Cartilha promoção da educação nutricional para Fenilcetonúricos.

\begin{tabular}{|l|l|}
\hline \multicolumn{1}{|c|}{ Conteúdo } & \multicolumn{1}{|c|}{ Apresentação } \\
\hline Definição e fisiopatologia da fenilcetonúria & Formato digital e interativo \\
Definição e fonte de proteínas & Parágrafos curtos, com frases completas \\
Alimentos com baixo e alto teor de & Imagens \\
proteínas & Tabelas \\
Tolerância à fenilalanina. & Infográficos \\
Teor de fenilalanina nos alimentos & \\
\hline
\end{tabular}

Os Cuidadores e familiares podem desempenhar um papel vital em manter sob tratamento seus parentes e amigos com fenilcetonúria. Assim, a cartilha, ao trabalhar também a educação nutricional com cuidadores e familiares, deixará clara a necessidade de envolvimento destas pessoas no tratamento e no planejamento de cuidados. Educar não é apenas doutrinar e transferir conteúdo, mas é oferecer uma experiência que prepare o indivíduo e seus cuidadores para a vida em sociedade com as restrições alimentares impostas pela doença. Estas pessoas são parceiras no apoio ao usuário do serviço, elas devem estar envolvidas no planejamento dos cuidados e ter suas próprias necessidades reconhecidas.

Materiais padronizados para educação contínua sobre a doença e seu tratamento, abrangentes, atraentes e independente da indústria são uma necessidade médica não atendida 
para pacientes adultos e adolescentes onde a adesão a dieta tende a diminuir devido a transição da responsabilidade pela saúde dos pais para os pacientes durante a adolescência e ao conhecimento limitado sobre doença e tratamento (MUNDY et al., 2002). Assim , o texto deve ser feito em parágrafos curtos, com frases completas, útil para instruções simples, com inclusão de imagens, tabelas e infográficos e para ajudar o usuário a entender o processo de cuidado. O texto pode ser construído de forma a antecipar e responder às perguntas do usuário, com uma estrutura de pergunta e resposta para incentivar o leitor a procurar informações.

O conceito de ISD é o processo de projetar aprendizagem por meio da instrução, onde o foco está na aprendizagem, e não no ensino. Esse conceito foi amplamente utilizado e aceito na área de educação como uma especialidade distinta. Os conceitos de Design Instrucional seguindo o modelo ADDIE será utilizado para o design e a gestão da construção desta cartilha educacional. O acrônimo ADDIE é uma sigla em inglês para: Analyze (Análise), Design, Develop (Desenvolvimento), Implement (Implementação) e Evaluate (Avaliação) (SENA et al., 2020).

O Modelo ADDIE é composto de 5 fases (BRANCH, 2009), as etapas da construção da cartilha seguem a estrutura abaixo:

1 - Análise, a primeira fase do Modelo ADDIE e, resumidamente, consiste na coleta de informações ou levantamento de necessidades. Nesta fase coletamos as informações para planejar a cartilha analisando: o conteúdo coletado da amostra dos pacientes para identificar o objetivo instrucional, os melhores formatos no Design. As principais expectativas e necessidades de informações e conteúdos sobre o tratamento da fenilcetonúria relatadas pelos pacientes está descrita no Quadro 02. Quarenta e sete pacientes realizam intervenção terapêutica para o tratamento da fenilcetonúria, em diálogo nas consultas 70,2\% (33/47) deles emitiram opinião sobre instrumento educacional, destes 45,5\% (15/33) consideraram que que o conhecimento atual que possui sobre a Fenilcetonúria e seu tratamento é bom. 93,9\% (31/33) acredita que é necessário ter uma Cartilha de consulta rápida sobre o Tratamento dietético da Fenilcetonúria. Em pergunta aberta, 66,7\% (22/33) opinaram que a cartilha deveria ter o teor de fenilalanina dos alimentos e dicas de alimentação na escola e no trabalho e 60,6\% (20/33) gostariam de saber sobre os alimentos permitidos e não permitidos. 
Quadro 02 - Resumo das expectativas e necessidades de informações e conteúdos sobre o tratamento da fenilcetonúria.

Explicações genéticas, sobre o porquê algumas crianças nascem com fenilcetonúria e outras da mesma família não.

Quantidade de fenilcetonúria nos alimentos in natura.

Opções de substituições dos alimentos e sugestões de cardápio.

Orientação sobre a dieta e as consultas.

Dicas de como melhorar a adesão com o complemento de aminoácidos sem FAL

Quantidade de fenilalanina nos alimentos industrializados.

2- Design, nesta fase devemos utilizar a criatividade para fazer o esboço da solução de educativa embasados na etapa anterior. O desenvolvimento de conteúdo baseado na literatura científica, conforme as necessidades apresentadas na etapa anterior. A literatura científica coletada nas bases de dados fornecerá bases sólidas para o desenvolvimento do conteúdo, para garantir sua confiabilidade. Pode ser feito um roteiro ou storyboard, onde as ideias sobre como pensar em abordar o conteúdo, a busca das melhores ilustrações e imagens. Nesta fase, é oportuno realizar a visita a bancos de imagens em sites (CTRLQ, Flickr, Image Raider, Google images, entre outros.) que serão acessados em busca das melhores ilustrações. As imagens didáticas serão selecionadas (Figura 01).
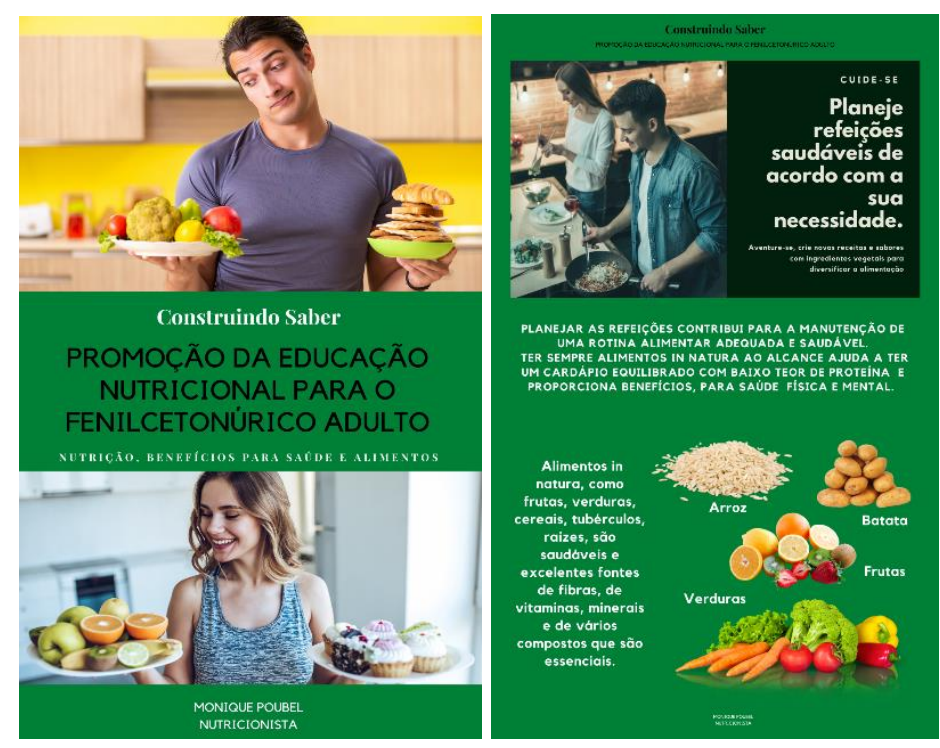
Figura 01 - Design e ilustração da cartilha - Construindo o Saber : Promoção da Educação Nutricional Para o Fenilcetonúrico Adulto. Fonte: Autoria própria (2021).

3 - Desenvolvimento, nesta fase será feira a criação da cartilha com a diagramação e editoração do conteúdo proporcionando facilidade de leitura e clareza de conteúdo. O conteúdo preliminar será selecionado e as informações consideradas essenciais, serão incluídas sendo submetidas a trabalhos de edição e diagramação. O desenvolvimento dessa fase baseia-se nos critérios previamente estabelecidos para todo o processo de construção da cartilha, ou seja, visual agradável, facilidade de leitura e clareza de conteúdo.

O roteiro de conteúdo indica a forma da disponibilização do conteúdo. Por exemplo, no caso de tópicos, serão abertos por abas que ao serem clicadas mostram seus conteúdos. Haverá sessões nos capítulos "Saiba mais" e, ao ser clicada, é exibida uma outra fonte bibliográfica ou informação adicional. Alguns trechos devem aparecer em uma formatação diferente indicando destaque, legislação, citação, dicas e exemplos.

4- Implementação, nesta fase a cartilha deve ser submetida à avaliação de especialistas, que irão realizar a validação da cartilha e haverá a decisão pelo ambiente virtual para acesso ao material produzido. $\mathrm{O}$ acesso pode ser feito em ambiente de acesso oficial de centros de referência responsáveis pelo cuidado de doenças raras ou seus órgãos responsáveis pela promoção e divulgação de ações educativas em saúde.

Os critérios adotados para a inclusão dos profissionais de saúde como especialistas devem ser atuar em serviços de doenças raras, ter experiência anterior em atividades de promoção da saúde e ter experiência relacionada à validação de materiais educativos e didáticos. Os especialistas devem avaliar a adequação e apresentação das informações, a facilidade de compreensão e o uso de vocabulário claro e objetivo; eles também indicarão possíveis erros conceituais, se houver. Em relação às imagens, os profissionais avaliaram a adequação da composição visual, a quantidade, atração e disposição. As sugestões de alterações serão incorporadas e será realizada uma nova edição de texto e diagramação.

5- Avaliação, nesta última etapa, deve ser feita uma entrevista durante a consulta de rotina com os pacientes para registros de observações sobre a cartilha, como o objetivo de identificar se os objetivos de aprendizagem foram alcançados e se materiais desenvolvidos atendeu os critérios previamente estabelecidos para todo o processo de construção da cartilha, ou seja, visual agradável, facilidade de leitura e clareza de conteúdo. Esta avaliação durante as consultas de rotina será feita com pacientes alfabetizados terão contato com a cartilha para 
validação quanto a compreensão do conteúdo, atração e facilidade de utilização da cartilha até que haja a ausência de novas recomendações de alterações.

Deve haver um link de interação com o autor, pois para as pessoas se coloquem no papel de protagonistas de suas escolhas e mudanças propondo mudanças no construto. É necessário motivação e informação sobre as possibilidades de escolhas alimentares, assim para a educação nutricional efetiva o produto deve possibilitar a abertura de espaço de diálogo, isto é necessário para a atualização do produto educativo com a realidade local, para que haja implementações periódicas.

A exigência de educação a distância na saúde com a pandemia de COVID-19 desafia todos os envolvidos nos processos de ensino aprendizagem com a necessidade de planejar, desenvolver e implementar instrumentos para o aprendizado à distância em formato digital. A importância do modelo de ADDIE para o planejamento deste produto educativo foi fundamental, pois possibilitou a identificação das necessidades, o conhecimento do públicoalvo, proposição de soluções para preencher as necessidades de educação em saúde e o mais importante, a avaliação dos resultados. A avaliação de resultados oferecerá a possibilidade de repensar objetivos e procedimentos utilizados para a elaboração do produto educacional.

\section{CONCLUSÃO}

Os materiais de educação do paciente são uma ferramenta importante para melhorar o conhecimento relacionado a doenças e tratamentos. Eles facilitam a comunicação entre a equipe médica e as crianças e adolescentes com doenças metabólicas e seus cuidadores. $\mathrm{O}$ ADDIE é mais que um modelo de design instrucional, é uma ferramenta de planejamento, demonstrado em forma de processo de tomada de decisões clareza e avaliação de objetivos ao longo da elaboração de um produto para educação nutricional para fenilcetonúricos.

Espera-se que essa inovação contribua positivamente para o aumento da habilidade e competência dos pacientes e cuidadores exercendo efeito prático na veiculação de informações de qualidade e pertinentes às necessidades dos fenilcetonúricos. Nesta iniciativa, está implícito um esforço para melhorar a qualidade de vida dos pacientes e da prestação do serviço de saúde. O desenvolvimento desta inovação é necessário para melhorar o conhecimento desta doença rara e ampliar de forma ágil a comunicação e a informação durante a pandemia de COVID-19. 
A alimentação é um dos pilares do tratamento da Fenilcetonúria e envolve valores afetivos, sensoriais e sociais. Para a efetividade deste tratamento, a alimentação restrita, saudável e adequada é imprescindível, ter informações lúdicas, simples e acessíveis sobre o tratamento é um dos pilares do empowerment. As ações de educação em saúde e nutrição realizadas devem expressar uma instrução voltada para o autocuidado, com conteúdo que desperte o olhar para si, proporcionando que o indivíduo possa ser um sujeito ativo no processo de aprendizagem sobre as escolhas alimentares para melhor efetividade do seu tratamento.

\section{REFERÊNCIAS}

BANNICK, A. A. et al. Outcomes of referrals to Child Protective Services for medical neglect in patients with phenylketonuria: Experiences at a single treatment center. Molecular Genetics and Metabolism, [S. l.], v. 115, n. 4, p. 151-156, 2015. Disponível em: https://doi.org/10.1016/j.ymgme.2015.06.003

BILDER, D. A. et al. Systematic Review and Meta-Analysis of Neuropsychiatric Symptoms and Executive Functioning in Adults With Phenylketonuria. Developmental Neuropsychology, [S. l.], v. 41, n. 4, p. 245-260, 2016. Disponível em: https://doi.org/10.1080/87565641.2016.1243109

BLAU, N. et al. DNAJC12 deficiency: A new strategy in the diagnosis of hyperphenylalaninemias. Molecular Genetics and Metabolism, [S. l.], v. 123, n. 1, p. 1-5, 2018. Disponível em: https://doi.org/10.1016/j.ymgme.2017.11.005

BLAU, N.; VAN SPRONSEN, F. J.; LEVY, H. L. Phenylketonuria. The Lancet, [S. l.], v. 376, n. 9750, p. 1417-1427, 2010. Disponível em: https://doi.org/10.1016/S01406736(10)60961-0.

BORRAJO, G. J. Panorama epidemiológico de la fenilcetonuria (PKU) em Latinoamérica. 2012. Acta Pediátrica México., [S. l.], v. 33, p. 279- 87, 2012. Disponível em: 
https://www.redalyc.org/articulo.oa?id=423640339001, 87.

BRANCH, R. M. Instructional Design: The ADDIE Approach. Boston, MA: Springer US, 2009. E-book. Disponível em: https://doi.org/10.1007/978-0-387-09506-6.

BRASIL. As Cartas da Promoção da Saúde / Ministério da Saúde. Secretaria de Políticas de Saúde.Projeto Promoção da Saúde. - Brasília: Ministério da Saúde, [S. l.], p. 56p, 2002.

BRASIL. Ministério da Saúde. Secretaria de Atenção à Saúde. Departamento de Atenção Básica. Orientações para a coleta e análise de dados antropométricos em serviços de saúde : Norma Técnica do Sistema de Vigilância Alimentar e Nutricional SISVAN / Ministério da. Brasil: [s. n.], 2011. Disponível em: http://bvsms.saude.gov.br/bvs/publicacoes/orientacoes_coleta_analise_dados_antropometrico s.pdf.

COOK, D. A. et al. Comparative effectiveness of instructional design features in simulationbased education: Systematic review and meta-analysis. Medical Teacher, [S. l.], v. 35, n. 1, p. e867-e898, 2013. Disponível em: https://doi.org/10.3109/0142159X.2012.714886

LITZKENDORF, S. et al. Use and importance of different information sources among patients with rare diseases and their relatives over time: a qualitative study. BMC Public Health, [S. l.], v. 20, n. 1, p. 860, 2020. Disponível em: https://doi.org/10.1186/s12889-02008926-9

MELO, B. C. P. de et al. Perspectivas sobre o uso das diretrizes de desenho instrucional para a simulação na saúde: revisão da literatura. Scientia Medica, [S. l.], v. 28, n. 1, p. 28852, 2018. Disponível em: https://doi.org/10.15448/1980-6108.2018.1.28852.

MUNDY, H. et al. Dietary control of phenylketonuria. The Lancet, [S. l.], v. 360, n. 9350, p. 
2076, 2002. Disponível em: https://doi.org/10.1016/S0140-6736(02)11959-3.

POLAND, B.; KRUPA, G.; MCCALL, D. Settings for Health Promotion: An Analytic Framework to Guide Intervention Design and Implementation. Health Promotion Practice, [S. l.], v. 10, n. 4, p. 505-516, 2009. Disponível em: https://doi.org/10.1177/1524839909341025.

SENA, B. dos S. et al. OVERWEIGHT AND ASSOCIATED FACTORS IN CHILDREN AND ADOLESCENTS WITH PHENYLKETONURIA: A SYSTEMATIC REVIEW. Revista Paulista de Pediatria, $[S . \quad$ l.], v. 38, 2020. Disponível em: https://doi.org/10.1590/1984-0462/2020/38/2018201. Acesso em: 1 set. 2020.

SINGH, R. H. et al. Recommendations for the nutrition management of phenylalanine hydroxylase deficiency. Genetics in Medicine, [S. l.], v. 16, n. 2, p. 121-131, 2014. Disponível em: https://doi.org/10.1038/gim.2013.179.

VAN WEGBERG, A. M. J. J. et al. The complete European guidelines on phenylketonuria: diagnosis and treatment. Orphanet Journal of Rare Diseases, [S. l.], v. 12, n. 1, p. 162, 2017. Disponível em: https://doi.org/10.1186/s13023-017-0685-2.

VOCKLEY, J., ANDERSSON, H. C., ANTSHEL, K. M., et al. Phenylalanine hydroxylase deficiency: diagnosis and management guideline. Genetics in Medicine, [S. l.], v. 16, n. 2, p. 188-200, 2014. Disponível em: https://doi.org/10.1038/gim.2013.157

ZELTNER, N. A. et al. Reducing complexity: explaining inborn errors of metabolism and their treatment to children and adolescents. Orphanet Journal of Rare Diseases, [S. l.], v. 14, n. 1, p. 248, 2019. Disponível em: https://doi.org/10.1186/s13023-019-1236-9. 
WATSON R. Instructional systems development. Washington DC: Institute of Education Science; 1981. 